\title{
Research on Media Coding of Dance Teaching Live System Based on iOS Platform
}

\author{
Chi Zhang \\ Basic Teaching and Research Institute, Bohai University, Jinzhou, P.R. China \\ 378290420@qq.com
}

Keywords: iOS platform; dance teaching; live system; audio coding; video coding

\begin{abstract}
In order to improve teaching informationization level of dance and promote the popularization of dance education, research the media coding of dance teaching live system based on iOS platform, support provide technical for the live system development. Firstly, study the iOS platform system structure constituted by cocoa touch, media application, core service, and sore OS; Secondly, study to capture the audio / video in the logic flow of live system in DirectShow; Then, begin the research of the audio coding technology of AAC, including the development process, the principle and the merit; Finally, the video coding technology of H.264 is studied, including video coding layer and network abstract layer. The results show that the coding technology has the features of high compression ratio, fast speed in encoding and decoding and so on.
\end{abstract}

\section{Introduction}

Dance is the most unique beauty of art forms in art aesthetic, both may flirt, and delta, time and space kneading, showing the harmony of rhythm, which is not only beautify the art of human action, but is much more than the appreciation of the beauty of body and soul. Dance education is the aesthetic way to show the image of art and unconsciously infect people, inspire and educate people. In order to improve people's ability of dance appreciation, it is necessary to popularize dance education. The popularity of the dance is mainly for the non - dance professional students and amateur dance enthusiasts, training the abilities in the dance aesthetic, dance training and basic performance, etc. $[1,2]$. Dance teaching uses the body with music, clothing, props, lighting and other artistic means, by the influence of time and space, cannot carry out in large-scale. The live mode of streaming media provides a new way for the popularization of dance education. Apple's mobile products as iPad, iPhone and iPod touch with high market share, this paper research the media coding of dance teaching live system based on the iOS platform, support provide technical for the live system development.

\section{iOS Platform Structure}

iOS is a mobile operating system developed by Apple Corp. Originally it is designed for iPhone, and later applied to touch iPod, iPad and TV Apple and other products. iOS is the same to Apple's OS X Mac operating system, which belong to the class of Unix commercial operating system. The interface, tools and resources of iOS programming are all contained in iOS SDK, through the integrated development environment finish the application development. The structure of iOS platform is showed in Fig.1.

In the four layer structure shows in Fig. 1, the Cocoa Touch Layer by key technologies such as the interface control, multi task, multi point touch, realizes human-machine interaction, touch operation application, provides a convenient method and technical support for the development of all types of intelligent terminal software; Media Application Layer, providing mutual combination which can complete various images, audio, video and other multimedia services in the application and development technology, bring the perfect multimedia experience for mobile devices; Core Service Layer, is located above the core OS layer to provide basic system services For all applications, provides the main framework, including CoreFoundation, CFNetwork, CoreLocation and security etc.; the Core OS Layer, is located in the bottom of the iOS hierarchy, including 
nuclear environment in operating system, drive and basic interface, by the $\mathrm{C}$ language to provide API function to complete the interaction with hardware and some other manipulation tasks [3-5].

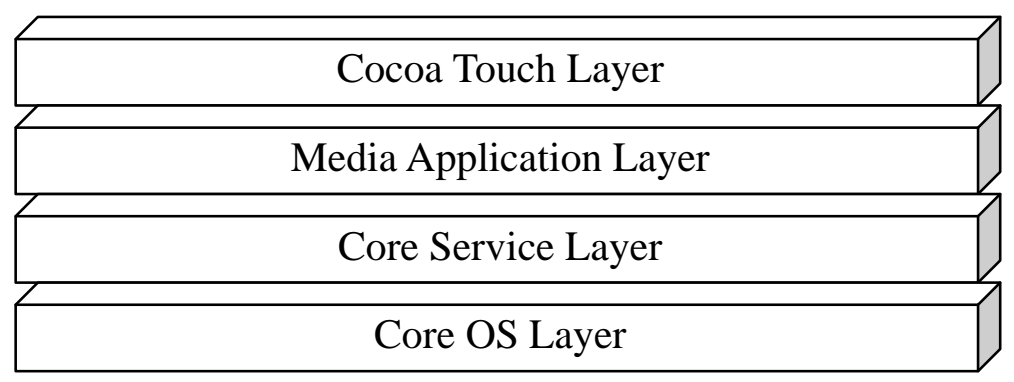

Fig. 1. iOS platform structure

\section{Live System Logical Process}

By the way of DirectShow capture audio / video, which is a set of development package on the windows platform streaming media processing, easily to capture data from acquisition card of driver model supported WDM, and the corresponding post processing and storage to a file. DirectShow also integrates other technologies of DirectX (for example DirectDraw, DirectSound), directly supports DVD player, video nonlinear editing, and data exchange with digital camera. The logical flow of the live system is shown in Fig. 2.

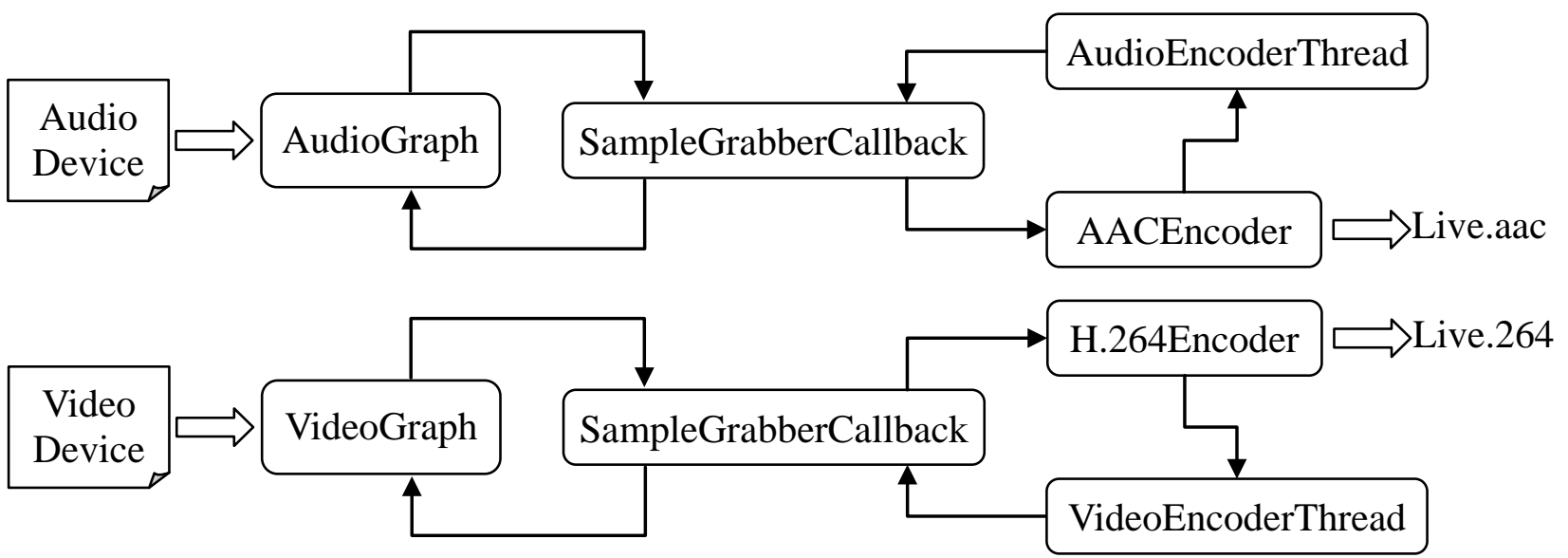

Fig. 2. Live system logical process

Video coding process is as follows: first of all initialize H.264 encoder, and then began to call DSVideoGraph in cycle, remove frames of the video from SampleGrabber, call H.264 to encoding, the process is relatively simple, frequency of calls is the video frame rate which want to get. Need to pay attention to is that H.264 coding is time-consuming, in the calculation of thread sleep time, to count the time consumed in the process, so as to avoid the acquisition of the video frame rate error.

The audio coding is the same as video coding process is also the initialization of the AAC encoder, and then loop call DS Audio Graph, remove the audio frame from the Sample Grabber, calling AAC encoding. The difference with the video is the frequency of the audio sample which is very fast, need to continue to collect, and SampleGrabber must capture the new data. 


\section{Audio Coding Technology}

Audio coding is to ensure the audio signal without distortion, in order to minimize the number of the original audio signal to facilitate limited storage or transmission of high audio quality audio information. The common audio coding mode includes waveform coding, parameter coding, mixed coding and perception coding, etc. For different audio coding methods, the operation complexity, the quality of the reconstructed signal, the compression rate, the coding and decoding delay are different, so it is suitable for different occasions.

AAC (Advanced Audio Coding) belongs to the perceptual coding, which is designed for file compression format of voice data, unlike MP3, use the new algorithm code, which is more efficient and more high "cost performance." The use of AAC format can make people feel more compact under the premise of the sound quality is not significantly reduced. AAC technological development process is showed in Fig. 3 [6].

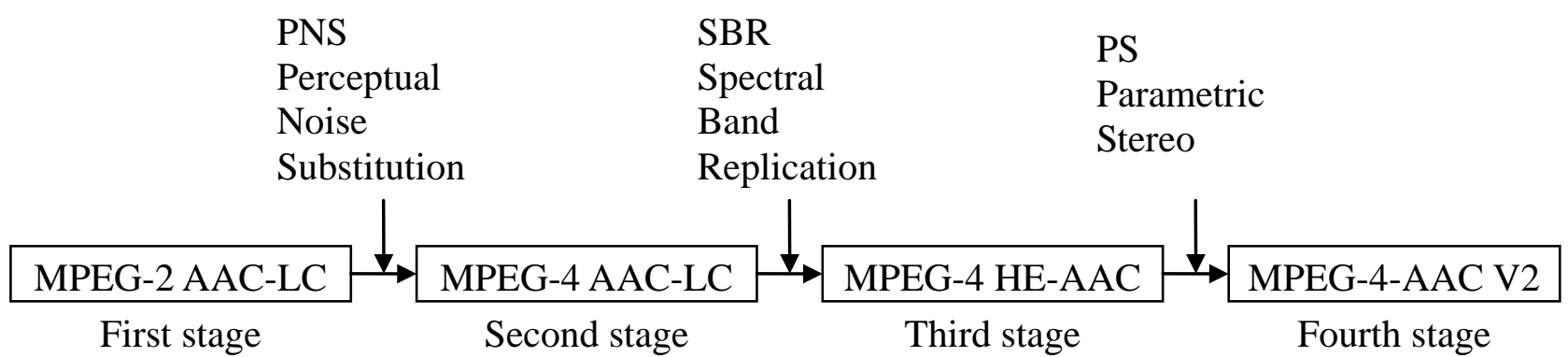

Fig. 3. Development process of AAC technology

HE-AAC V2 is by the three kinds of technology of MPEG-4 audio standard technology of AAC (Advanced Audio Coding), SBR (spectral band replication) and PS (parametric stereo), with the formation of a new audio coding method. MPEG-4 AAC provides a kind of method can be as good as all audios material in encoding and decoding, like other MPEG audio encoding methods, the AAC also analysis the energy components of audio signal and the use of the "masking effect" of the human ear, in order to find component can be other sound mask on hearing from the audio signal. MPEG-4 SBR is a band expansion of tools, and the original audio encoder joint working for broadening the bandwidth of the audio, the audio encoder to half the bit rate transmission with the quality of the audio signal. MPEG-4 PS is a compression coding method for stereo audio signal, and provides a new method for improving the efficiency of low rate stereo coding.

\section{Video Coding Technology}

In the video live application, the video quality and the network bandwidth occupy is the contradiction, the higher the video stream occupies bandwidth is, the higher the video quality is. So if the quality of the video effect is high, the network bandwidth becomes large. The key to solve this problem is the video coding and decoding technology.The structure of H.264 is divided into two layers from function and arithmetic, which is VCL (Video Coding Layer) and Network (Abstraction Layer NAL), as shown in Fig. 4. 


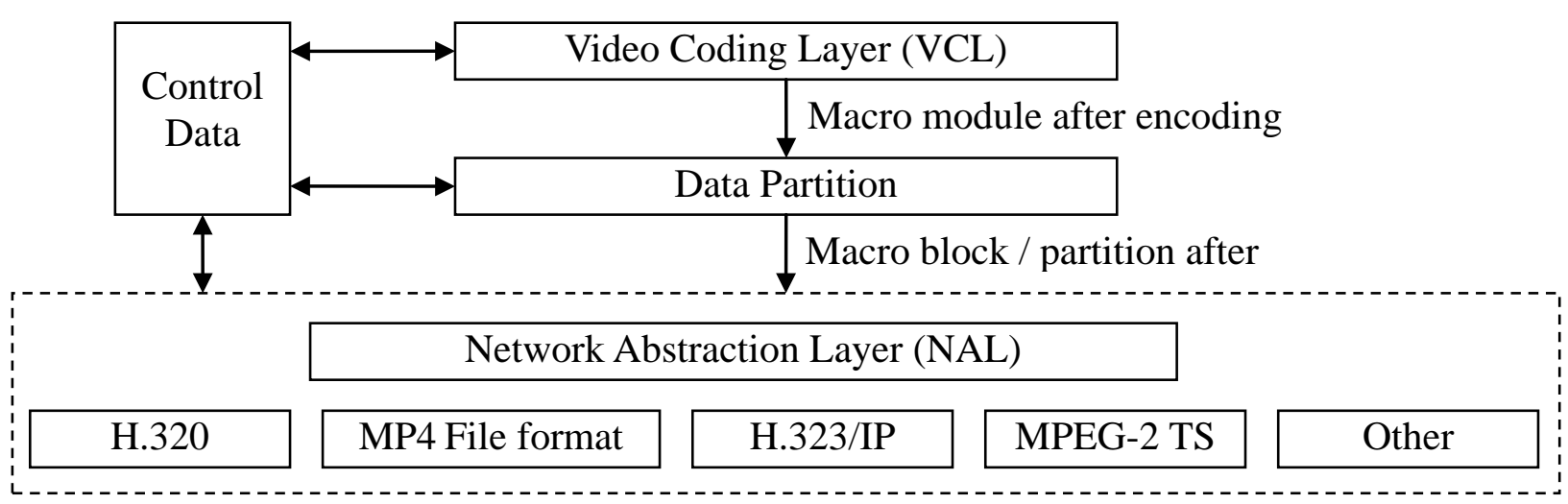

Fig .4. Hierarchical structure of H.264

VCL is responsible for the compression of high efficiency video coding. The hybrid coding framework based on block motion compensation, transformation coding and entropy coding adopted. The processing object is block and macro block data. VCL is the core of video coding, including many tools for error recovery, and a lot of advanced video coding techniques are adopted to improve the coding efficiency.

The NAL layer takes the NCL unit as the basic data format, not only contains all the video information, but also provides the transmission layer or the storage media information. The task of NAL is to provide the appropriate mapping method to map the head information and data to the transmission protocol, and can eliminate the overhead of group frame and repeat synchronization in packet switching. In order to improve H.264, NAL customization VCL data format ability in the different characteristics of network, between the NAL and VCL defined based on packet interface, packaging, and the corresponding signaling also belong to the part of VAL [7-9].

\section{Conclusion}

Quality education is a kind of education mode which is aimed at improving the overall quality of the education, and dancing education is one of the effective ways to promote the quality education. The use of video broadcast mode to achieve the popularization of dance education, can improve the artistic quality of the students and the people, aesthetic taste, psychological quality and aesthetic quality [10]. iOS platform uses streaming media transmission protocol and the real-time media data transmission mechanism to reduce the workload of the server and the client. Using the media encoding method is developed for the dance teaching live system, with high compression ratio, high quality and smooth voice and image, which has important significance to improve the informatization of dance education teaching and promote the dance art popularity.

\section{References}

[1] Y. Jiang, "Study on the connotation of dance art: Research on four aspects of dance appreciation, teaching, creation and protection," Master's degree of Harbin Normal University, 2013.

[2] Y. H. Sun, " How to cultivate college students dance appreciation ability," Music Magazine, vol. 10, no. 3, pp. 172, 2014.

[3] C. Liu, Q. Zhu, Kenneth A. Holroyd, Elizabeth K. Seng, "Status and trends of mobile-health applications for iOS devices: A developer's perspective," Journal of Systems and Software, vol. 84, no. 11, pp. 2022-2033, 2011.

[4] Jonathan Zdziarski, "Identifying back doors, attack points, and surveillance mechanisms in iOS devices," Digital Investigation, vol. 11, no. 8, pp. 3-19, 2014.

[5] P. Lou, X. P. Pang, S. T. Ai, "Development of Polar Mobile Information Platform Basec on Android and iOS," Chinese Journal of Polar Research, vol. 27, no. 6, pp. 98 - 103, 2015. 
[6] Y. C. Zhang, G. R. Hu, "Coding theory and its application of HE-AAC V2," Chinese cable TV, vol. 14, no. 3, pp. 293 - 296, 2006.

[7] Mithilesh Kumar Jha, Sumantra Dutta Roy, Brejesh Lall, "DEMD-based video coding for textured videos in an H.264/MPEG framework," Pattern Recognition Letters, vol. 51, no.6, pp. 30 - 36, 2015.

[8] Y. D. Wu, Y. Sun, Z. D. Feng, et al., "A novel total variation based frame layer rate control algorithm for H.264/AVC," Journal of Visual Communication and Image Representation, vol. 25, no. 5, pp. 879-890, 2014.

[9] D. W. Xu, R. D. Wang, Y. Q. Shi, "An improved reversible data hiding-based approach for intra-frame error concealment in H.264/AVC," Journal of Visual Communication and Image Representation, vol. 25, no.2, pp. 410 - 422, 2014.

[10] B. Yu, "College dance teaching and college students art quality education," Education and Vocation, vol. 98, no. 3, pp. 183-184, 2014. 